\section{Mortality among mental health services for older adults during the COVID-19 pandemic: a retrospective analysis from South London}

During the COVID-19 pandemic, older people with dementia and/or living in care homes had disproportionately high mortality rates (Suárez-González et al., 2020). However, to our knowledge, no study has investigated mortality within a mental health service for older adults (MHOA) covering both inpatient and outpatient settings.

We carried out a retrospective analysis of mortality in recent MHOA service users covering the first 10 months of 2020 and comparing these to similar period in 2019 using data from the South London and Maudsley NHS Foundation Trust (SLaM) Biomedical Research Centre (BRC) Case Register. SLaM is the sole specialist mental healthcare provider for 1.2 million residents in a Southeast London catchment including services for older adults and those with dementia, and the database has approval for secondary analysis (18/SC/0372). As a comparison, we obtained publicly available mortality data from the Office for National Statistics (ONS), for people of all ages for the SLaM Southeast London catchment area. Death within MHOA was defined if the patient was under MHOA at the time of death or if death happened within 30 days following discharge from MHOA.

We defined eleven 4-week periods from the beginning of week 1 to the end of week 44 of 2019 and 2020. We then determined the number of deaths and calculated the relative risk (RR) of mortality in each 4-week period in 2020 (exposure period for COVID-19) compared with the same period in 2019 (unexposed period for COVID-19) for MHOA service users as well as for the SLaM catchment population.

In total 7,148 people died in the first 44 weeks of 2020 within the SLaM catchment, of whom 418 (5.8\%) were SLaM MHOA patients. The most striking spike in mortality risk for MHOA patients occurred during weeks 13-16 (March 23-April 19, 2020), while the general population had a significantly increased risk of death in weeks 13-16 (March 23-April 19, 2020) and 17-20 (April 20May 17, 2020) compared to 2019 (see Figure 1). Other than in the catchment population, no increased $R R$ was detected in MHOA service users after April 20, 2020. Risk of death was neither increased nor decreased between weeks $21-44$ in both the catchment and MHOA service user populations.

Our results suggest that the COVID-19 pandemic might have affected MHOA service users severely in its early phase.

While some of our findings can be explained by higher age and comorbidities, care home residence could also have played a role. It is known that $26.3 \%$ of all COVID-19-related deaths occurred in care home residents in England during 2020 and dementia and Alzheimer's disease were common (26\%) pre-existing conditions cited for deaths involving COVID-19 (ONS, 2020). Patients with dementia and cognitive impairment may have had limited access to accurate information on the pandemic and, especially in the early stages, might not have been able to follow recommendations to reduce the spread of COVID-19 (Wang et al., 2020). While age increases the risk of a more severe course of COVID-19 (Mok et al., 2020), the underlying neurodegenerative process of dementia could also be implicated. APOE $€ 4$ has been associated with an increased COVID-19-related hospitalization risk and, mechanistically, might be linked with increased cytokine production in response to inflammatory stimuli, which could intensify the already aggressive inflammatory response associated with COVID-19, resulting in a so-called cytokine storm (Mok et al., 2020).

In conclusion, despite their higher age, which is usually associated with smaller social networks (English and Carstensen, 2014) and consequently a lower viral infection risk, mortality in users of MHOA services increased rapidly in the beginning of the pandemic. Hence, this is a group that needs special protection early in a pandemic situation.

\section{Conflicts of interest}

RS has received recent research funding from Roche, Janssen, GSK, and Takeda. GP, CM, $\mathrm{MB}$, and LV declare no conflict of interest.

\section{Source of funding}

The data resource, GP, CM, and RS are partfunded by the National Institute for Health Research (NIHR) Biomedical Research Centre at South London and Maudsley NHS Foundation Trust and King's College London, and RS by the National Institute for Health Research (NIHR) Applied 


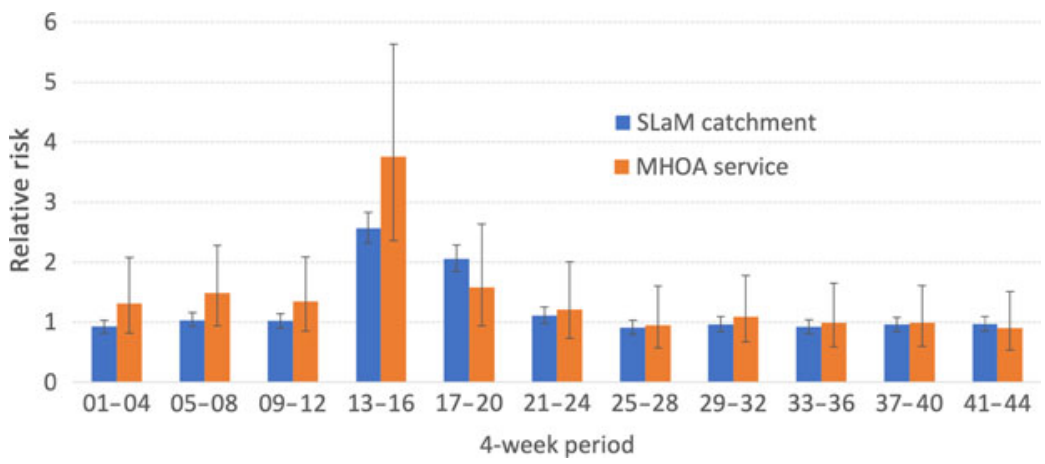

Figure 1. Relative risk of deaths in 2020 compared with year 2019 in 4-weekly intervals in MHOA service users and the catchment area general population. $\mathrm{MHOA}=$ Mental Health of Older Adults. SLaM catchment $=$ geographical area receiving mental health and dementia care from South London and Maudsley NHS Foundation Trust.

Research Collaboration South London (NIHR ARC South London) at King's College Hospital NHS Foundation Trust. The views expressed are those of the authors and not necessarily those of the NHS, the NIHR, or the Department of Health.

\section{Description of authors' roles}

The study was conceived by LV and RS. Analyses were carried out by GP and CM. The manuscript was written by GP and finalized by CM and with substantial text contribution from all authors.

\section{Data sharing statement}

No additional data are available.

\section{References}

English, T. and Carstensen, L. L. (2014). Selective narrowing of social networks across adulthood is associated with improved emotional experience in daily life. International fournal of Behavioral Development, 38, 195-202.

Mok, V. C. T. et al. (2020). Tackling challenges in care of Alzheimer's disease and other dementias amid the
COVID-19 pandemic, now and in the future. Alzheimers \& Dementia, 16, 1571-1581.

ONS (2020). Deaths Involving COVID-19, England and Wales: Deaths Occurring in fune 2020. Statistical Bulletin, p. 28. Office for National Statistics.

Suárez-González, A. et al. (2020). Impact and mortality of COVID-19 on people living with dementia updated 19th August 2020. International Long-term Care Policy Network ILPN, 31.

Wang, H. et al. (2020). Coronavirus epidemic and geriatric mental healthcare in China: how a coordinated response by professional organizations helped older adults during an unprecedented crisis. International Psychogeriatrics, 32, 1117-1120.

Gayan Perera, ${ }^{1, \dagger}$ Christoph Mueller, ${ }^{1,2, \dagger^{(D}}$ Matthew Broadbent, ${ }^{2}$ Robert Stewart ${ }^{1,2}$ and LATHA VELAYUdHAN ${ }^{1,2}$

${ }^{1}$ King's College London, Institute of Psychiatry, Psychology and Neuroscience, London, UK

${ }^{2}$ South London and Maudsley NHS Foundation Trust, London, UK

Correspondence should be addressed to: Latha Velayudhan, M6.01, Old Age Psychiatry Department, Institute of Psychiatry, Psychology and Neuroscience, Denmark Hill, UK. Email: latha.velayudhan@kcl.ac.uk.

$\dagger$ These authors are co-first authors who have worked together and contributed equally. 